\title{
Compact Switched-Beam Antenna Employing a Four-Element Slot Antenna Array for Digital Home Applications
}

\author{
Ming-Iu Lai, Student Member, IEEE, Tzung-Yu Wu, Jung-Chin Hsieh, Chun-Hsiung Wang, and \\ Shyh-Kang Jeng, Senior Member, IEEE
}

\begin{abstract}
In this paper a compact switched-beam antenna is proposed. The antenna is composed of a four-element antenna array based on L-shaped quarter-wavelength slot antenna elements. Such an antenna element is a planar structure and presents a directional radiation pattern in the azimuth plane. Its maximum radiation direction is toward near the direction of the open end of the slot. As a result, the open ends of the four slot antennas are arranged toward $0, \pi / 2, \pi$, and $3 \pi / 2$, respectively. The statuses of these antennas are controlled by some diodes. Consequently, by carefully controlling the diodes, an antenna with several switchable patterns can be achieved. To prove the concept, a 2.4-2.5 GHz switched-beam antenna for WLAN applications is designed and implemented. Its size is $52 \mathrm{~mm}$ in square. The antenna possesses eight directional patterns and many nearly omnidirectional patterns in the azimuth plane. The experiment results fully demonstrate the performance of the proposed design. The envelope correlations and the characteristics of the designed antenna are also discussed. Due to the compact size and low manufacture cost, such a design can be a promising solution for digital home applications to overcome multipath problems and increase the transmission data rate.
\end{abstract}

Index Terms-Antenna array, correlation, slot antenna.

\section{INTRODUCTION}

W HILE MORE and more digital contents are transmitted using wireless technologies, the transmission data rate plays a critical role in the way to digital home. Suffering from multipath fadings, the data rate drops drastically as transmission distance increases in indoor environments. Antennas with

Manuscript received September 4, 2007; revised March 19, 2008. Published September 4, 2008 (projected). This work was supported in part by ASUSTeK Computer Inc., Taiwan, R.O.C. and in part by the National Science Council of Taiwan, R.O.C., under Grant NSC95-2221-E-002-092.

M.-I. Lai is with the Graduate Institute of Communication Engineering, National Taiwan University, Taiwan, R.O.C. and also with the Core Technology Center, Strategic Investment Headquarter, ASUSTeK Computer Inc., Taiwan, R.O.C. (e-mail: milai1102@yahoo.com.tw).

T.-Y. Wu is with the Graduate Institute of Communication Engineering, National Taiwan University, Taiwan, R.O.C.

J.-C. Hsieh was with the Graduate Institute of Communication Engineering, National Taiwan University, Taiwan, R.O.C. He is now with the Core Technology Center, Strategic Investment Headquarter, ASUSTeK Computer Inc., Taiwan, R.O.C.

C.-H. Wang is with the Core Technology Center, Strategic Investment Headquarter, ASUSTeK Computer Inc., Taiwan, R.O.C.

S.-K. Jeng is with the Graduate Institute of Communication Engineering and the Department of Electrical Engineering, National Taiwan University, Taiwan, R.O.C. (e-mail: skjeng@ew.ee.ntu.edu.tw).

Digital Object Identifier 10.1109/TAP.2008.928775 switchable beam or various diversities are well-known solutions to overcome this problem [1]-[7].

A large number of studies on switched-beam antennas can be found in literatures [8]-[17]. Such antennas are one solution to provide high and robust transmission rate in rich multipath environments by focusing its main beam toward the direction of the desired signal. Traditionally, Butler matrix was used to design a switched-beam antenna [8]-[11]. However, integrating an antenna array with a Butler matrix would occupy a large volume. A switched-beam disc antenna was proposed by Graziano [12], offering promising features such as wide bandwidth and beam steering over $360^{\circ}$ in the azimuth plane, but suffered from the bulky three-dimensional structure and presented difficulties in integrating with wireless devices in home. A compact six-sector antenna employing three intersecting dual-beam microstrip Yagi-Uda arrays was proposed by Naoki [13]. This antenna could achieve large size reductions. The penalty was high manufacture cost since six single-pole-three-throw (SP3T) switches were used in the design.

Some reconfigurable antennas offered switchable beam features [14]-[17]. A reconfigurable annular slot antenna was proposed by Nikolaou [14]; however, an additional matching network was needed to improve its performance. Reconfigurable spiral microstrip antennas were proposed by Huff [15] and Chang [16], where RF MEMS switches were applied to reconfigure the spiral. But the thickness of the substrate was chosen to be a quarter guided-wavelength at the operation frequency. It was suitable only for high-frequency designs. A pattern reconfigurable microstrip parasitic array was proposed by Zhang [17]. The antenna had a conformal profile, small size and simple structure but its beam cannot steer over $360^{\circ}$ in the azimuth plane.

Switched-beam antennas for digital home applications must be low profile and price and require steering over $360^{\circ}$ in the azimuth plane. The problems of conventional designs are high manufacture cost, complicated design procedures and bulky three-dimensional structures. In this paper a planar, compact and switched-beam antenna is proposed. The use of planar technology to implement antennas presents many advantages like potential low cost and easy to assembly. The proposed antenna is mainly composed of a four-element antenna array based on the L-shaped slot antenna element [18]-[22]. Such an antenna element presents a directional radiation pattern in the azimuth plane. Hence, we arrange the antennas with maximum radiation direction toward different directions. The details will be given later in the paper. 


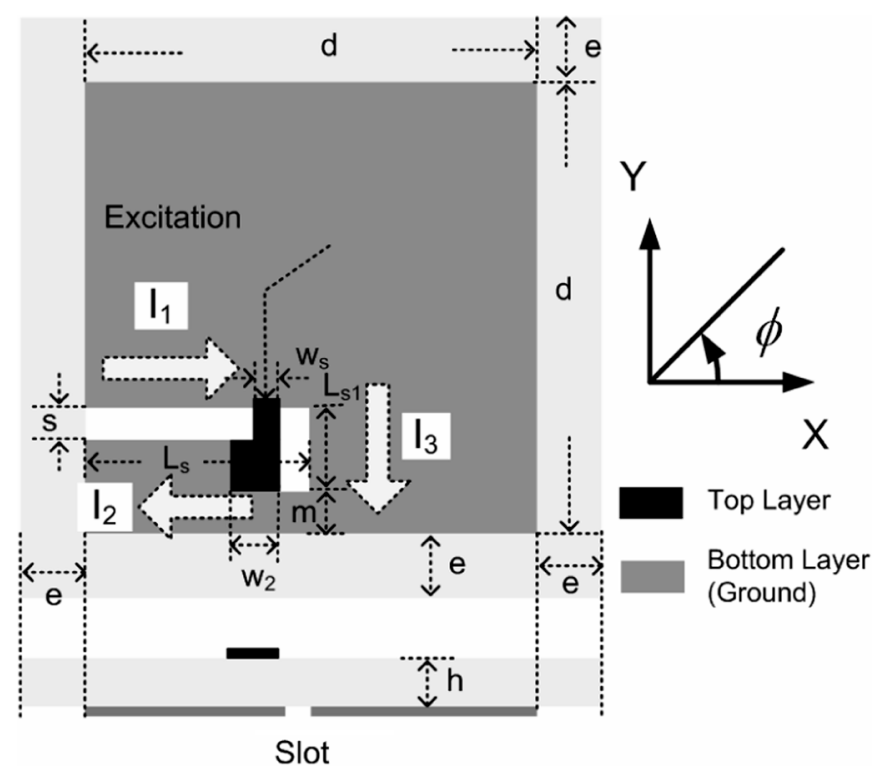

Fig. 1. Geometry of a conventional L-shaped slot antenna. The antenna lies in the $x y$-plane where $\mathrm{s}=1 \mathrm{~mm}, \mathrm{~L}_{\mathrm{s}}=16.5 \mathrm{~mm}, \mathrm{w}_{2}=3 \mathrm{~mm}, \mathrm{w}_{\mathrm{s}}=0.8$ $\mathrm{mm}, \mathrm{L}_{\mathrm{s} 1}=3 \mathrm{~mm}, \mathrm{~m}=2 \mathrm{~mm}, \mathrm{e}=6 \mathrm{~mm}, \mathrm{~d}=40 \mathrm{~mm}, \mathrm{~h}=0.8 \mathrm{~mm}$, and $\varepsilon_{r}=4.4$.

This paper is organized as follows. The design concept will be described in Section II, where radiation properties of the antenna element and theory of operation of the proposed antenna are presented. To prove the concept, a $2.4-2.5 \mathrm{GHz}$ switchedbeam antenna for WLAN applications is designed and implemented. The simulation and measurement results are exhibited in Section III. Next, some discussions on the resultant antenna are given in Section IV. Finally, brief conclusions are summarized in Section V.

\section{ANTENNA DESIGN}

\section{A. Antenna Element}

In order to explain the proposed antenna array, the radiation property of the antenna element used in the array must be introduced firstly. The geometry of the conventional L-shaped quarter-wavelength slot antenna [18]-[22] is shown in Fig. 1. The antenna is designed to be resonant at $2.45 \mathrm{GHz}$, where the length of the slot is about a quarter guided-wavelength of the slotline. At the resonant frequency, the induced current distribution on the ground plane is roughly plotted in Fig. 1.

The currents may be simply decomposed into three parts, named $I_{1}, I_{2}$, and $I_{3}$. The antenna radiations are mainly contributed by $I_{3}$ because the directions of $I_{1}$ and $I_{2}$ are opposite and the radiations are partially canceled by each other. Accordingly, the patterns are like those of a small dipole oriented in the $y$-axis, leading that the gain pattern $G_{\phi}$ in the $x y$-plane and $G_{\theta}$ in the $y z$-plane are bidirectional, while $G_{\phi}$ in the $x z$-plane presents a nearly omnidirectional radiation. Furthermore, owing to the shape of the ground plane, $G_{\phi}$ in the $x y$-plane becomes somewhat directional and the maximum radiation direction is toward near the direction of the open end of the slot. Different from the other field components, $G_{\phi}$ in the $y z$-plane is attributed to the currents $I_{1}$ and $I_{2}$ since the direction of $I_{3}$ is perpendicular

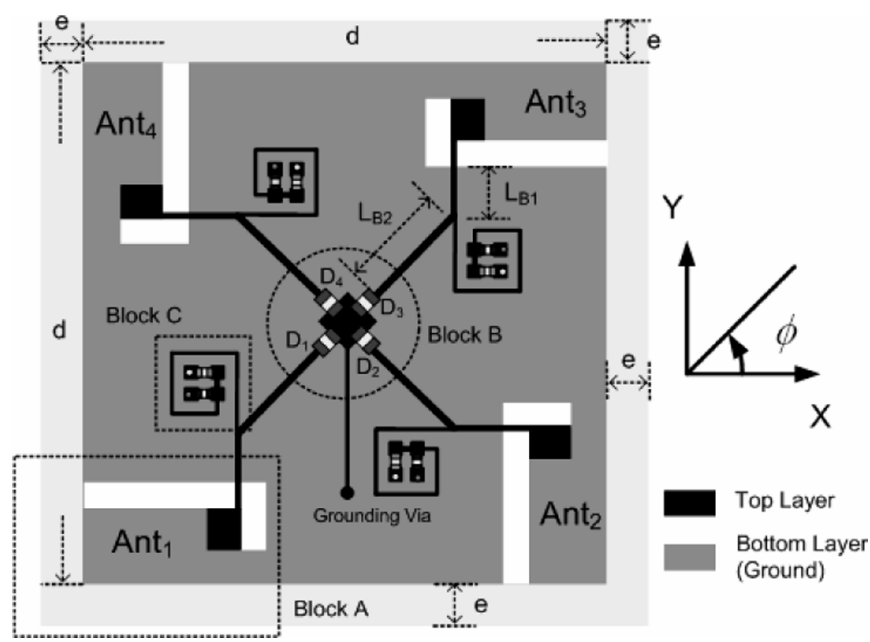

Fig. 2. Structure of the proposed antenna array. The detailed structure of Block A is shown in Fig. 1 and those of Block B and C in Fig. 3.

to the $\phi$-direction in the $y z$-plane. Therefore the $G_{\phi}$ pattern has minima at antenna's broadside directions. The simulated peak gain is $-1.2 \mathrm{dBi}$ and occurs in the $x y$-plane.

As discussed above, the L-shape slot antenna presents a directional pattern in the azimuth plane. For a single antenna system, a directional pattern is capable of alleviating multipath effects but such a pattern may have problems in communication when the desired signal comes from the directions of the nulls. In this paper the design concept is to combine several such directional antennas to form a compact switched-beam antenna.

\section{B. Antenna Description}

The structure of the proposed antenna is depicted in Fig. 2, where the detailed structures are plotted in Figs. 1 and 3. Basically, this antenna is a four-element array with radiation elements based on the L-shaped slot antenna. The reasons for the antenna arrangement will be explained later. The array is fed by a $50 \Omega$ SMA connector at its center point and excited through a vertical probe. There are two reasons for this design. The first is to maintain a geometrical symmetry of the basic structure; the other is to keep the feeding network as far as possible away from the radiation elements in order to reduce undesired interactions between them. For the purpose of biasing networks design, the antenna feeding point is grounded using a quarter-wavelength long short-circuited microstrip stub.

The four pin diodes in Fig. 2 are designed as a single-polefour-throw (SP4T) switch, simpler and cheaper than using a commercial SP4T switch. The four transmission lines connected between the diodes and the antenna elements are equal in length and width. Because a diode appears inductive in ON-state, the matching is accomplished by applying a square metal patch on the excitation probe, as shown in Fig. 3(a). To bias the diode and guarantee isolation between the biasing circuit and the antenna, a quarter-wavelength long high impedance microstrip with a grounded capacitor is designed, as shown in Fig. 3(b). Near the capacitor, a resistor is used to limit the current flowing into the diode. Thus, while applying a high voltage on a control pin, the diode will be in forward biasing, enabling the connected antenna. 

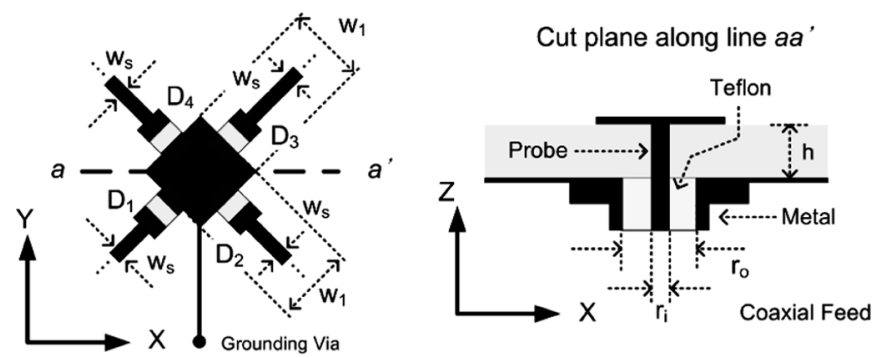

(a)
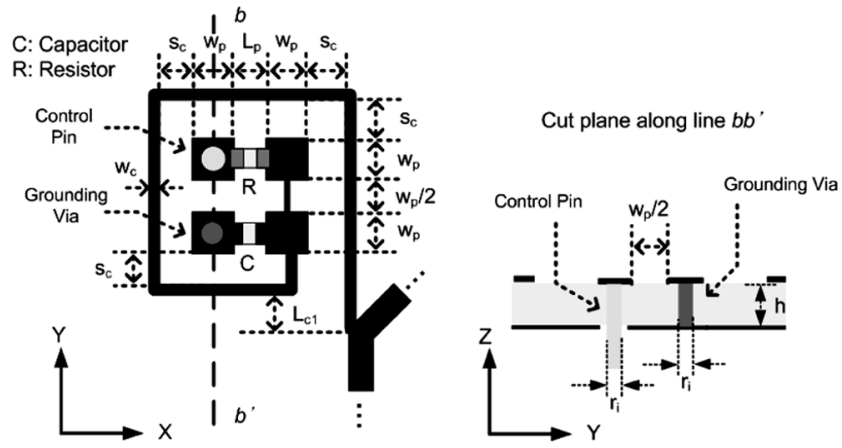

(b)

Fig. 3. Detailed structures of the proposed antenna. (a) Block B in Fig. 2. (b) Block C in Fig. 2.

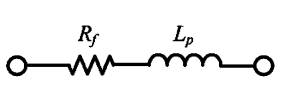

(a)

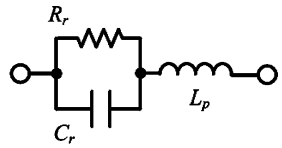

(b)
Fig. 4. Equivalent circuit models of a PIN diode in forward and reverse biasing, where $R_{f}$ is the forward resistance, $L_{p}$ is the lead inductance, $R_{r}$ is the reverse parallel resistance, and $C_{r}$ is the diode capacitance. (a) Forward biasing. (b) Reverse biasing.

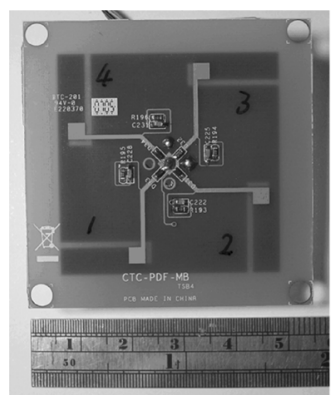

(a)

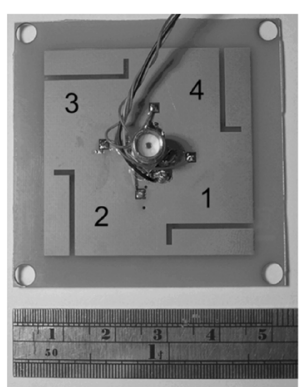

(b)
Fig. 5. Photographs of the designed antenna. (a) Top view. (b) Bottom view.

\section{Theory of Operation}

As mentioned above, the L-shaped slot antenna presents a directional radiation pattern in the azimuth plane and its maximum radiation direction is toward near the direction of the open end of the slot. As a result, the open ends of the four slot antennas are arranged toward $0, \pi / 2, \pi$, and $3 \pi / 2$, respectively. Assume that the equivalent antenna spacing is small enough compared to the wavelength of the operation frequency so that the phase variation between antenna elements from far-field observation

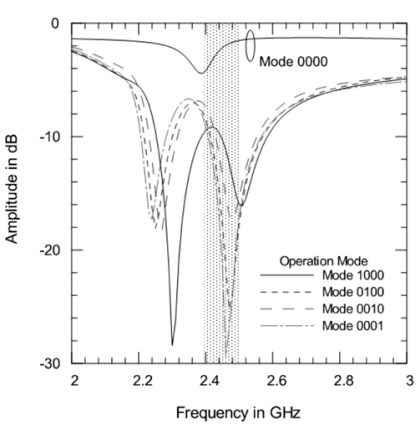

(a)

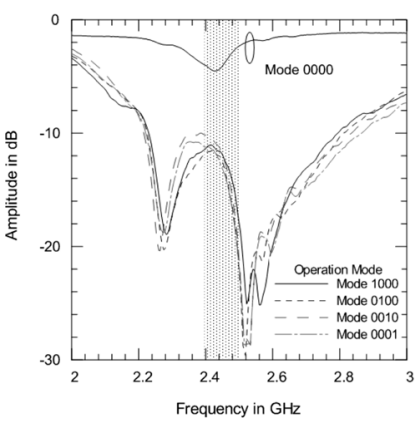

(c)

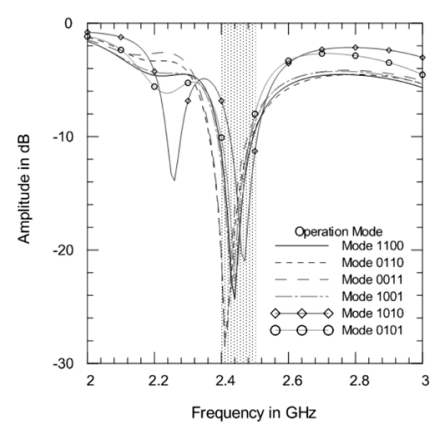

(b)

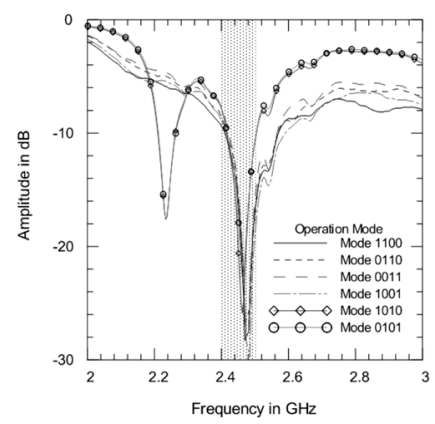

(d)
Fig. 6. Simulated and measured reflection coefficients. (a) and (b) are the simulated results. (c) and (d) are the measured results.

TABLE I

Detailed PARAMETERS OF THE DESIGNED ANTENNA

\begin{tabular}{cccccc}
\hline Parameters & Value & Parameters & Value & Parameters & Value \\
\hline $\mathrm{er}$ & 4.4 & $\mathrm{~W}_{1}$ & 3.0 & $\mathrm{~s}$ & 1.0 \\
$\mathrm{~h}$ & 0.8 & $\mathrm{~W}_{2}$ & 1.0 & $\mathrm{~s}_{\mathrm{c}}$ & 0.5 \\
$\mathrm{~L}_{\mathrm{s}}$ & 16.5 & $\mathrm{~W}_{\mathrm{c}}$ & 0.125 & $\mathrm{e}$ & 6.0 \\
$\mathrm{~L}_{\mathrm{s} 1}$ & 3.0 & $\mathrm{~W}_{\mathrm{p}}$ & 1.0 & $\mathrm{r}_{\mathrm{o}}$ & 4.1 \\
$\mathrm{~L}_{\mathrm{c} 1}$ & 1.0 & $\mathrm{~W}_{\mathrm{s}}$ & 0.8 & $\mathrm{r}_{\mathrm{i}}$ & 1.0 \\
$\mathrm{~L}_{\mathrm{p}}$ & 1.0 & $\mathrm{~d}$ & 40.0 & $\mathrm{~L}_{\mathrm{B} 1}$ & 9.0 \\
$\mathrm{~L}_{\mathrm{B} 2}$ & 4.0 & $\mathrm{R}$ & $1000 \mathrm{hms}$ & $\mathrm{C}$ & $100 \mathrm{pF}$ \\
$\mathrm{Vc}$ & 3.0 Volt & & & & \\
\hline
\end{tabular}

Unit: $\mathrm{mm}$

can be neglected. Intuitively, while one of the antennas is active, the pattern will be shifted toward the direction $n \pi / 2$, where $n=0,1,2$, or 3 ; while two adjacent antennas are active, the pattern will be shifted toward the direction $(1+2 n) \pi / 4$, where $n=0,1,2$, or 3 . For the diagonal antenna pairs, the patterns will be nearly omnidirectional since the patterns of the antennas in a pair are almost complementary in the azimuth plane. Then, four diodes are designed to control these antenna elements. Consequently, by carefully controlling these diodes, a switched-beam antenna with eight directional patterns and two nearly omnidirectional patterns can be achieved.

To fulfill the above assumption, the equivalent antenna spacing should be much smaller than the wavelength of the operation frequency. In practical designs, small antenna spacing may induce strong mutual couplings and the couplings will distort antenna patterns. Thus, while designing the width of the ground plane, which determines the equivalent antenna spacing, the level of coupling should be considered carefully. 

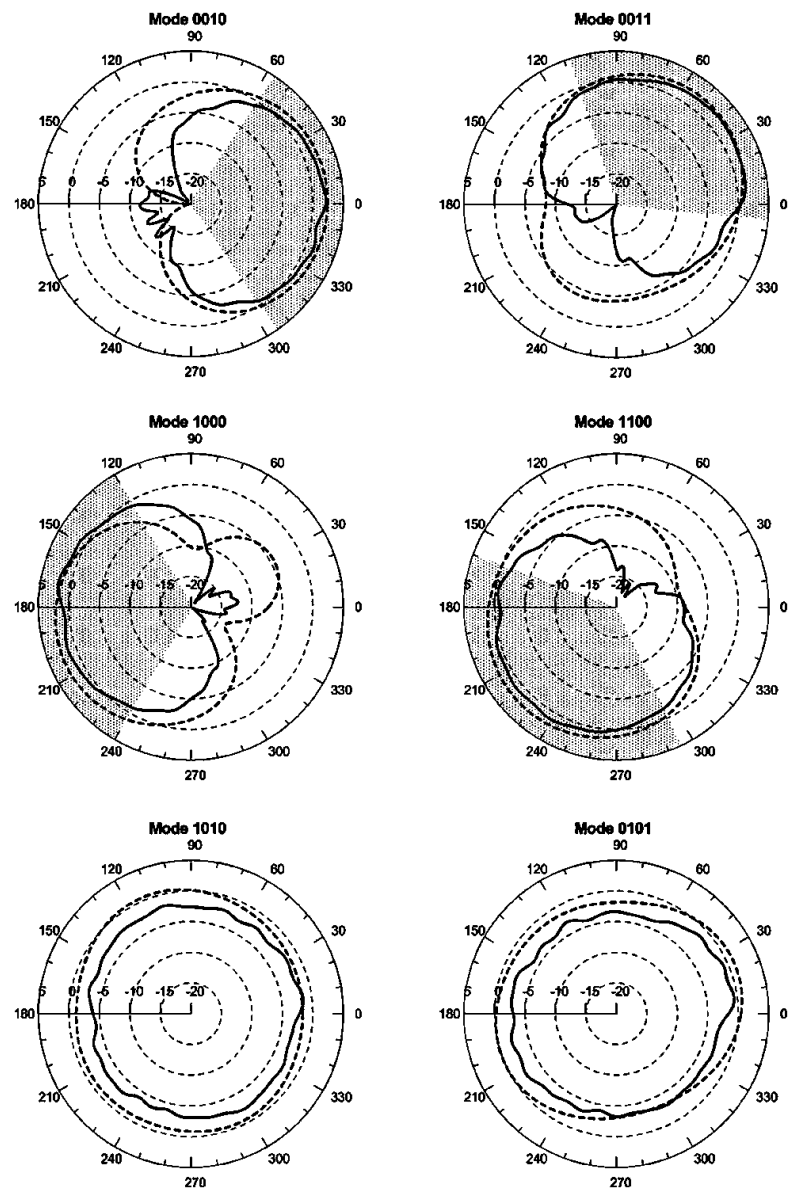
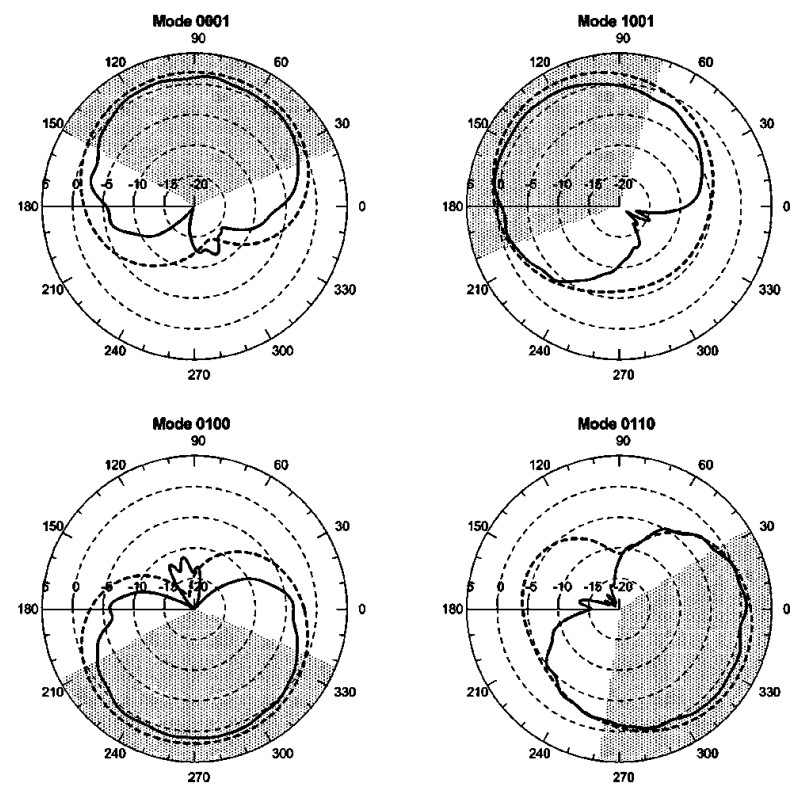

Measurement

Simulation

Fig. 7. Simulated and measured gain patterns $G_{\phi}$ versus $\phi$ in decibels in the $x y$-plane at $2.45 \mathrm{GHz}$.

\section{Simulation AND MEASUREMENT RESUltS}

To prove the concept, a $2.4-2.5 \mathrm{GHz}$ switched-beam antenna for WLAN applications is designed using an FR4 substrate with thickness $0.8 \mathrm{~mm}$. Infineon BAR64-02 V PIN diodes are used in this work, with forward resistance $2.1 \mathrm{Ohms}$, reverse parallel resistance $3000 \Omega$, diode capacitance $0.17 \mathrm{pF}$, and lead inductance $0.6 \mathrm{nH}$. The reflection coefficients were measured by Agilent 8364B network analyzer at National Taiwan University and the gain patterns by SATIMO SG64 3-D anechoic chamber at ASUSTeK.

The antenna was modeled using Ansoft HFSS software. To obtain precise results, the equivalent circuits of PIN diode in both forward and reverse biasings, as shown in Fig. 4, should be considered during simulations. In HFSS, the elements in the equivalent circuits can be modeled by lumped RLC boundary conditions.

The photographs of the designed antenna are included in Fig. 5. The overall size of the antenna is $52 \mathrm{~mm}$ in square, and the detailed design parameters are listed in Table I. Note that the holes at the corners in Fig. 5 are used to fix the antenna. In this work the width of ground plane, $d$, is chosen to be $40 \mathrm{~mm}$ corresponding to $0.327 \lambda_{0}$ at $2.45 \mathrm{GHz}$. The equivalent spacing between adjacent antennas will be smaller than $0.327 \lambda_{0}$. The simulated maximum coupling between antenna elements is
$-15 \mathrm{~dB}$. Fig. 6 provides the simulated and measured reflection coefficients, where the shaded portions represent the bandwidth of interest. In the legend, mode 1000 denotes $D_{1}$ in ON-state and the others in OFF-state, mode 1100 denotes $D_{1}$ and $D_{2}$ in ON-state and the others in OFF-state, and so on. It can be seen that the measured results agree well with the simulated results, and the reflection coefficients are smaller than $-10 \mathrm{~dB}$ within the bandwidth of interest. For mode 0000, the small dip around $2.4 \mathrm{GHz}$ is due to the diode capacitance in OFF-state. Additionally, some operation modes of the designed antenna are resonant at around $2.2 \mathrm{GHz}$, resulting from interactions between the antenna elements and the feeding and biasing networks. However, this effect can be ignored in realistic applications since there is a pre-selected bandpass filter to reject out-of-band signals in conventional RF front-end circuits.

Note that the control lines and batteries are carefully placed along the coaxial cable so that they do not affect the radiation pattern much. Fig. 7 exhibits the simulated and measured gain patterns $G_{\phi}$ in the $x y$-plane at $2.45 \mathrm{GHz}$, where the shadings indicate the region of half power radiation plotted according to the measured patterns. As expected, the radiation beam changes clearly, and the main beam portions in the measured and simulated patterns match pretty well. The slight discrepancies in the opposite directions of the main beams may mainly come from the coaxial cable, control lines and batteries, located under 
TABLE II

PATTERN CHARACTERISTICS OF THE DESIGNED ANTENNA

\begin{tabular}{ccccccccc}
\hline \multirow{2}{*}{ Mode } & $\begin{array}{c}\text { Maximum Beam Direction } \\
\text { (Degrees) }\end{array}$ & \multicolumn{2}{c}{$\begin{array}{c}\text { 3dB Beam Width } \\
\text { (Degrees) }\end{array}$} & \multicolumn{2}{c}{$\begin{array}{c}\text { Front-To-Back Ratio } \\
\text { (dB) }\end{array}$} & \multicolumn{2}{c}{$\begin{array}{c}\text { Antenna Gain } \\
\text { (dBi) }\end{array}$} \\
\cline { 2 - 9 } & Measured & Simulated & Measured & Simulated & Measured & Simulated & Measured & Simulated \\
\hline 0010 & 2 & 8 & 114 & 140 & 15.2 & 8.1 & 2.1 & 2.4 \\
0011 & $\mathbf{4 0}$ & 49 & 114 & 121 & 9.3 & 6.4 & 2.0 & 2.4 \\
0001 & $\mathbf{8 4}$ & 92 & 128 & 159 & 10.4 & 7.5 & 1.4 & 1.9 \\
1001 & 134 & 126 & 126 & 125 & $\mathbf{9 . 7}$ & 7.5 & 1.5 & 2.8 \\
1000 & $\mathbf{1 7 0}$ & 202 & 120 & 114 & 11.1 & 7.0 & $\mathbf{2 . 1}$ & 2.6 \\
1100 & $\mathbf{2 4 0}$ & 226 & 134 & 158 & 10.2 & 5.4 & 1.3 & 2.3 \\
0100 & $\mathbf{2 7 8}$ & 266 & $\mathbf{1 3 0}$ & 153 & $\mathbf{1 4 . 4}$ & 7.1 & $\mathbf{1 . 2}$ & 2.0 \\
0110 & $\mathbf{3 2 0}$ & 329 & $\mathbf{1 3 0}$ & 118 & $\mathbf{9 . 7}$ & 7.0 & $\mathbf{1 . 3}$ & 2.3 \\
1010 & - & - & - & - & - & - & -1.4 & 0.5 \\
0101 & - & - & - & - & - & - & $-\mathbf{0 . 5}$ & 1.0 \\
\hline
\end{tabular}

the antenna and used to bias the diodes in the resultant antenna. They are not taken into account during simulations.

The pattern characteristics are summarized in Table II. The maximum beam directions are listed in the first major column, revealing that the beams change regularly. The second major column is half power beam width (HPBW). The average measured and simulated HPBWs are 124.5 and 136.0 degrees, respectively. The third major column lists the front-to-back (F/B) ratio defined by the ratio of the directivity of the maximum lobe in the range of \pm 60 degrees to that of the opposite direction [13], showing that the average measured and simulated $\mathrm{F} / \mathrm{B}$ values are 11.3 and $7.0 \mathrm{~dB}$, respectively.

The last major column lists the antenna gains in the $x y$-plane. The measured gains are slightly lower than the simulated ones, mainly resulting from the PIN diodes. During simulations, the forward resistance of diode is set to be $2.1 \Omega$ according to the data sheet. However, this value was measured at $100 \mathrm{MHz}$. The exact resistance will be larger than $2.1 \Omega$ at microwave frequencies, causing the measured gains inferior to the simulated ones.

\section{DISCUSSIONS}

\section{A. Envelope Correlations}

Diversity gain is an important indicator for the improvement from a diversity antenna system relative to a single antenna one, which depends on envelope correlations and power imbalance between the diverted signals [2]. A general form of envelope correlation can be calculated as [23], [24]

$$
\rho_{e}^{i j} \approx \frac{\left|\oiint \bar{e}^{i}(\theta, \phi) \cdot \bar{e}^{j^{*}}(\theta, \phi) d \Omega\right|^{2}}{\oiint\left|\bar{e}^{i}(\theta, \phi)\right|^{2} d \Omega \oiint\left|\bar{e}^{j}(\theta, \phi)\right|^{2} d \Omega}
$$

where $d \Omega=\sin \theta d \theta d \phi, \bar{e}^{i}(\theta, \phi)$ is the radiated electric field of the $i$ th operation mode and the asterisk denotes complex conjugate. If the correlation is high, the received signals may suffer from deep fading simultaneously. Papers [23] and [24] show that
TABLE III

ENVELOPE CORRELATIONS BETWEen THE OPERATION MODES OF THE DESIGNED ANTENNA

\begin{tabular}{cccccccccc}
\hline Mode & 0010 & 0011 & 0001 & 1001 & 1000 & 1100 & 0100 & 0110 \\
\hline 0010 & 1.000 & 0.594 & 0.197 & 0.106 & 0.080 & 0.108 & 0.258 & 0.688 \\
0011 & 0.594 & 1.000 & 0.657 & 0.307 & 0.372 & 0.013 & 0.026 & 0.191 \\
0001 & 0.197 & 0.657 & 1.000 & 0.774 & 0.304 & 0.072 & 0.001 & 0.007 \\
1001 & 0.106 & 0.307 & 0.774 & 1.000 & 0.404 & 0.359 & 0.060 & 0.003 \\
1000 & 0.080 & 0.372 & 0.304 & 0.404 & 1.000 & 0.517 & 0.220 & 0.091 \\
1100 & 0.108 & 0.013 & 0.072 & 0.359 & 0.517 & 1.000 & 0.712 & 0.223 \\
0100 & 0.258 & 0.026 & 0.001 & 0.060 & 0.220 & 0.712 & 1.000 & 0.560 \\
0110 & 0.688 & 0.191 & 0.007 & 0.003 & 0.091 & 0.223 & 0.560 & 1.000 \\
\hline
\end{tabular}

the criteria $\rho_{e}<0.5$ is necessary to obtain an obvious improvement from a diversity system. Table III shows the correlations according to the simulated radiation electric fields for the modes with directional patterns, where the shaded portions indicate the values smaller than 0.5 . It can be seen that the most correlations meet the criteria and few are larger than 0.5 . The high correlation occurs when the difference of the maximum beam directions of two modes is about 45 degrees. The way to reduce the value is to increase the spacing between antenna elements. However, it will enlarge the antenna size.

To develop a diversity system based on a switched-beam antenna, a cost-effective way is via a switched combining scheme. Such a system switches between operation modes when the received signal strength is lower than a predefined threshold. Table III brings important information while designing a switching process. Since the patterns of modes 1010 and 0101 are nearly omnidirectional, these modes can be applied to detect dynamically a proper threshold value or used to broadcast messages. Suppose one of the operation modes with directional patterns is active, it will keep working until the 
TABLE IV

Comparison Between the Proposed Antenna and the Traditional Antennas

\begin{tabular}{|c|c|c|c|c|c|c|c|}
\hline \multirow{2}{*}{ Ref. } & \multirow{2}{*}{$f_{0}(\mathrm{GHz})$} & \multicolumn{2}{|c|}{ Substrate } & \multirow{2}{*}{$\begin{array}{l}\text { Normalized } \\
\text { size }\end{array}$} & \multirow{2}{*}{$\begin{array}{c}\text { Measured } \\
\text { Antenna Gain } \\
\text { (dBi) }\end{array}$} & \multirow{2}{*}{$\begin{array}{c}\text { Worst Measured } \\
\text { Bandwidth }(\mathrm{GHz}) \\
\left(\left|\mathrm{S}_{11}\right|<-10 \mathrm{~dB}\right)\end{array}$} & \multirow{2}{*}{$\begin{array}{l}\text { Number of } \\
\text { Modes }\end{array}$} \\
\hline & & $\operatorname{Er}(\tan \delta)$ & $\mathrm{h}(\mathrm{mm})$ & & & & \\
\hline [13] & 5.0 & $2.2(0.0008)$ & 1.6 & $1.83 \lambda_{0}$ diameter & $>10$ & $4.70 \sim 5.15$ & 6 \\
\hline [14] & 3.65 & 2.2 (NA) & 6.35 & $\sim 0.5 \lambda_{0}{ }^{*} 0.5 \lambda_{\mathrm{g}}$ & $\sim 0$ & $3.67 \sim 3.87$ & 3 \\
\hline [15] & 6.85 & $2.2(0.0008)$ & 3.175 & $\sim 0.5 \Lambda_{0}$ in square & $\sim 0$ & $6.78 \sim 6.87$ & 2 \\
\hline [16] & 5.8 & $6.15(0.0025)$ & 0.635 & $\sim \lambda_{0}$ in square & NA & NA & 3 \\
\hline [17] & 10.0 & $3.27(0.004)$ & 3.175 & $\sim 0.6 \Lambda_{0}$ in square & $4-6$ & NA & 2 \\
\hline This Work & 2.45 & $4.4(0.02)$ & 0.8 & $0.43 \lambda_{0}$ in square & $-0.5 \sim 2.1$ & $2.42-2.51$ & $8+$ \\
\hline
\end{tabular}

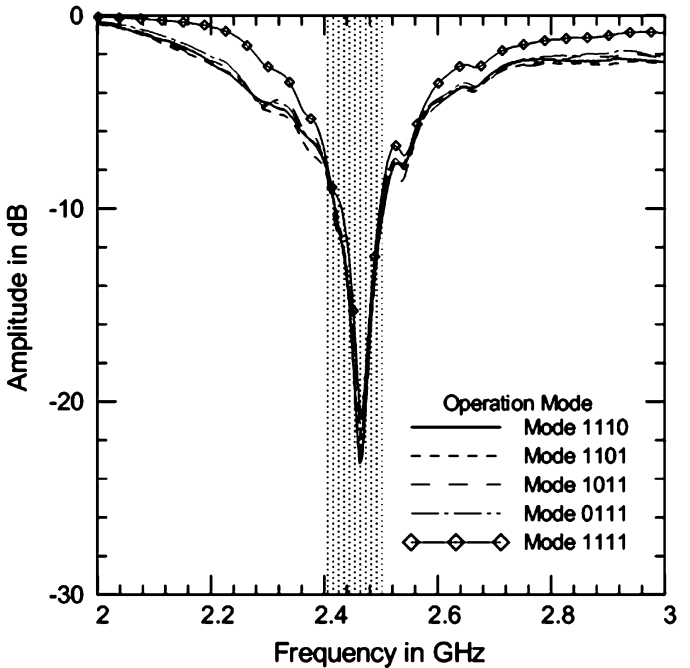

(a)

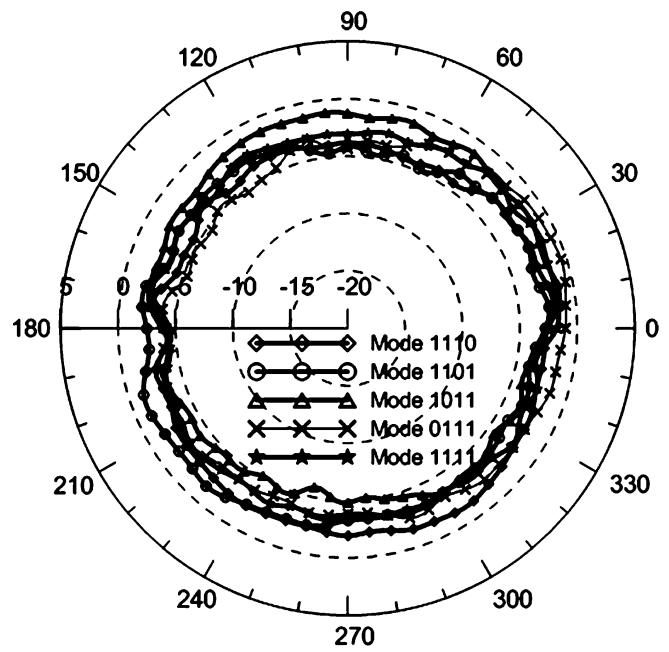

(b)

Fig. 8. Measured reflection coefficients and gain patterns $G_{\phi}$ in $\mathrm{dBi}$ in the $x y$-plane for modes $1110,1101,1011,0111$, and 1111.

received signal strength goes below the determined threshold. When the strength is detected below the threshold, another mode is selected such that the correlation between the new and the original modes is lower than 0.5. By using this simple strategy, a good diversity system can be achieved.

\section{B. Other Operation Modes}

So far, the performance of the designed antenna has been presented. There are five modes remained except mode 0000. Their performances are investigated here. Fig. 8 shows the measured reflection coefficients and gain patterns in the $x y$-plane. It can be seen that the reflection coefficients are below $-8.0 \mathrm{~dB}$ within the bandwidth of interest and their patterns are nearly omnidirectional. From a system design consideration, these modes can be discarded since their characteristics are similar to those of modes 1010 and 0101 .

\section{Pros and Cons}

According to previous discussions, the reflection coefficients of the proposed design for most operation modes are lower than $-10 \mathrm{~dB}$ within the bandwidth of interest, and the most correlations meet the criteria. Compared with the traditional switchedbeam antennas [8]-[17], the proposed antenna is a cost-effective solution since it composes of a common FR4 substrate and several cheap lumped elements rather than expansive microwave substrates and switches. Table IV lists the comparison between the proposed antenna and the published planar switched-beam antennas [13]-[17], showing that it is more compact than traditional ones. Moreover, the proposed antenna has more than eight operation modes. However, due to its compact size, the use of PIN diodes, and the lossy substrate, the proposed antenna suffers from a lower antenna gain. According to Table II, the maximum gain is $2.1 \mathrm{dBi}$.

The use of RF-MEMS switches may provide an alternative solution to PIN diodes due to their high-Q property. Though MEMS devices present many problems [25], the use of MEMS devices may be a good solution to improve the antenna gain in the future.

\section{CONCLUSION}

A planar, compact, and switched-beam antenna has been proposed. The design concept for the proposed antenna has also been presented. To prove the concept, a $2.4-2.5 \mathrm{GHz}$ switchedbeam antenna for WLAN applications was designed and implemented successfully. The experiment results fully demonstrated the performance of the proposed antenna. The envelope correlations and the characteristics of the designed antenna were also investigated. 
The switched-beam antenna has the potential to be applied on an adaptive multiple input multiple output (MIMO) system to enhance channel capacity and robustness of the traditional MIMO wireless communication systems. This application will be further investigated and tested in the near future. Due to the miniature size and low manufacture cost, the proposed antenna can be a promising solution for digital home applications.

\section{ACKNOWLEDGMENT}

The authors would like to thank the Associate Editor and the anonymous reviewers for their helpful comments. They also wish to express their gratitude to Mr. Z.-J. Yan, Mr. R. Hong, and Mr. R. Ko, EMI Department, ASUSTeK Computer Inc., for their help in antenna measurement. Also, they are further indebted to Dr. T.-M. Parng, CTO, ASUSTeK Computer Inc., for his great and constant support on this work.

\section{REFERENCES}

[1] C. B. Dietrich, K. Dietze, J. R. Nealy, and W. L. Stutzman, "Spatial, polarization, and pattern diversity for wireless handheld terminals," IEEE Trans. Antennas Propag., vol. 49, no. 9, pp. 1271-1281, Sep. 2001

[2] S. R. Saunders, Antenna and Propagation for Wireless Communication System. New York: Wiley, 1999, ch. 15.

[3] A. Alexiou and M. Haardt, "Smart antenna technologies for future wireless systems: Trends and challenges," IEEE Commun. Mag., pp. 90-96, Sep. 2004.

[4] B. A. Cetiner, H. Jafarhani, J. Y. Qian, H. J. Yoo, A. G. , and F. De Flaviis, "Multifunctional reconfigurable MEMS integrated antennas for adaptive MIMO systems," IEEE Commun. Mag., pp. 62-70, Dec. 2004.

[5] S. Smith and D. V. Thiel, Switched Parasitic Antennas for Cellular Communications. Boston, MA: Artech House, 2002.

[6] R. Vaughan, "Switched parasitic elements for antenna diversity," IEEE Trans. Antennas Propag., vol. 47, no. 2, pp. 309-405, Feb. 1999.

[7] N. L. Scott, M. O. Leonard-Taylor, and R. G. Vaughan, "Diversity gain from a single-port adaptive antenna using switched parasitic elements illustrated with a wire and monopole prototype," IEEE Trans. Antennas Propag., vol. 47, no. 6, pp. 1066-1070, Jun. 1999.

[8] D. Parker and D. C. Zimmermann, "Phased arrays-Part II: Implementations, applications, and future trends," IEEE Trans. Microw. Theory Tech., vol. 50, no. 3, pp. 688-698, Mar. 2002.

[9] M. Bona, Manholm, J. P. Starski, and B. Svensson, "Low-loss compact Butler matrix for a microstrip antenna," IEEE Trans. Microw. Theory Tech., vol. 50, no. 9, pp. 2069-2075, Sep. 2002.

[10] M. Nedil, T. A. Denidni, and L. Talbi, "Novel butler matrix using CPW multilayer technology," IEEE Trans. Microw. Theory Tech., vol. 54, no. 1, pp. 499-507, Jan. 2006.

[11] M. Barba, J. E. Page, J. A. Encinar, and J. R. Montejo-Garai, "A switchable multiple beam antenna for GSM-UMTS base stations in planar technology," IEEE Trans. Antennas Propag., vol. 54, no. 11, pp. 3087-3094, Nov. 2006.

[12] G. Cerri, R. D. Leo, V. M. Primiani, C. Monteverde, and P. Russo, "Design and prototyping of a switching beam disc antenna for wideband communications," IEEE Trans. Antennas Propag., vol. 54, no. 12, pp. 3721-3726, Dec. 2006.

[13] N. Honma, T. Seki, K. Nishikawa, K. Tsunekawa, and K. Sawaya, "Compact six-sector antenna employing three intersecting dual-beam microstrip Yagi-Uda arrays with common director," IEEE Trans. Antennas Propag., vol. 54, no. 11, pp. 3055-3062, Nov. 2006.

[14] S. Nikolaou, R. Bairavasubramanian, C. Lugo, I. Carrasquillo, D. C. Thompson, G. E. Ponchak, J. Papapolymerou, and M. M. Tentzeris, "Pattern and frequency reconfigurable annular slot antenna using PIN diodes," IEEE Trans. Antennas Propag., vol. 54, no. 2, pp. 439-448, Feb. 2006.

[15] G. H. Huff and J. T. Bernhard, "Integration of packaged RF MEMS switches with radiation pattern reconfigurable square spiral microstrip antennas," IEEE Trans. Antennas Propag., vol. 54, no. 2, pp. 464-469, Feb. 2006
[16] C. W. Jung, M. J. Lee, G. P. Li, and F. De Flaviis, "Reconfigurable scan-beam single-arm spiral antenna integrated with RF-MEMS switches," IEEE Trans. Antennas Propag., vol. 54, no. 2, pp. 455-463, Feb. 2006.

[17] S. Zhang, G. H. Huff, and J. T. Bernhard, "A pattern reconfigurable microstrip parasitic array," IEEE Trans. Antennas Propag., vol. 52, no. 10, pp. 2773-2776, Oct. 2004.

[18] M. I. Lai, T. Y. Wu, J. C. Hsieh, C. H. Wang, and S. K. Jeng, "A miniature, Planar, and switched-beam smart antenna employing a four-element slot antenna array for digital home applications," in Proc. IEEE Int. Symp. Antenna Propag., Jun. 2007.

[19] S. K. Sharma, L. Shafai, and N. Jacob, "Investigate of wide-band microstrip slot antenna," IEEE Trans. Antennas Propag., vol. 52, no. 3, pp. 865-872, Mar. 2004.

[20] S. I. Latif, L. Shafai, and S. K. Sharma, "Bandwidth enhancement and size reduction of microstrip slot antennas," IEEE Trans. Antennas Propag., vol. 53, no. 3, pp. 994-1003, Mar. 2005.

[21] A. P. Zhao and J. Rahola, "Quarter-wavelength wideband slot antenna for 3-5 GHz mobile applications," IEEE Antennas Wireless Propag. Lett., vol. 4, pp. 421-424, 2005.

[22] W. S. Chen and K. Y. Ku, "Broadband design of a small non-symmetric ground $\lambda / 4$ open slot antenna," Microw. J., vol. 50, no. 1, pp. 110-121, Jan. 2007.

[23] R. G. Vaughan and J. B. Andersen, "Antenna diversity in mobile communications," IEEE Trans. Veh. Technol., vol. VT-36, pp. 149-172, Nov. 1987.

[24] M. Karaboikis, C. Soras, G. Tsachtsiris, and V. Makios, "Compact dual-printed inverted-F antenna diversity systems for portable wireless devices," IEEE Antennas Wireless Propag. Lett., vol. 3, pp. 9-14, 2004.

[25] G. M. Rebeiz, RF MEMS Theory, Design, and Technology. New York: Wiley, 2003, ch. 1.

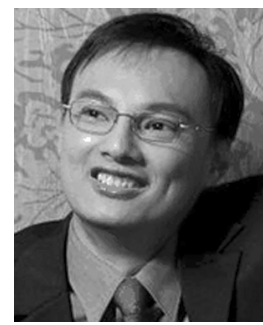

Ming-Iu Lai (S'04) was born in Kaohsiung, Taiwan, R.O.C., in 1976. He received the B.S.E.E. degree from National Taiwan University of Science and Technology, Taipei, Taiwan, R.O.C., in 1998, and the M.S.E.E. degree from National Taiwan University, Taipei, Taiwan, R.O.C., where he is currently working toward the Ph.D. degree.

In 2006, he joined ASUSTeK Computer Inc., Taiwan, R.O.C., where he is now a Researcher. His current research interest includes reconfigurable antenna and smart antenna designs.

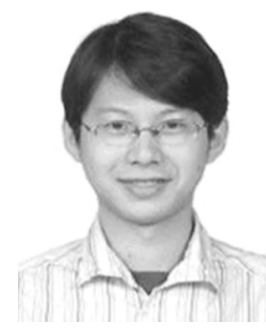

Tzung-Yu Wu was born in Pingtung, Taiwain R.O.C, in 1983. He received the B.S. degree from National Tsing Hua University, Hsinchu, Taiwan, R.O.C., in 2006. He is currently working toward the M.S.E.E. degree at National Taiwan University, Taipei, Taiwan, R.O.C. sign.

His current research interest is patch antenna de-

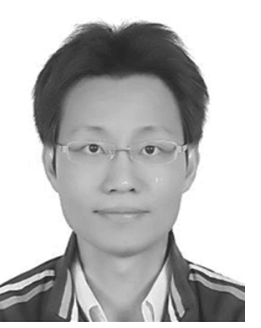

Jung-Chin Hsieh was born in Kaohsiung, Taiwan, R.O.C., in 1980. He received the B.S. degree from National Cheng Kung University, Tainan, Taiwan, R.O.C., in 2002 and the M.S. degree from the Graduate Institute of Communication Engineering, National Taiwan University, Taiwan, R.O.C., in 2007.

In 2007, he joined ASUSTeK Computer Inc., Taiwan, R.O.C., where he is now an Engineer. 


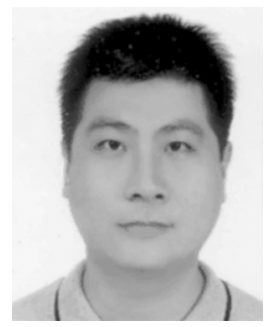

Chun-Hsiung Wang was born in Taipei, Taiwan, R.O.C., in 1964. He received the B.S. degree from Vanung University, Tao-Yuan, Taiwan, R.O.C., in 1998.

In 2005, he joined ASUSTeK Computer Inc., Taiwan, R.O.C., where he is now a Senior Engineer.

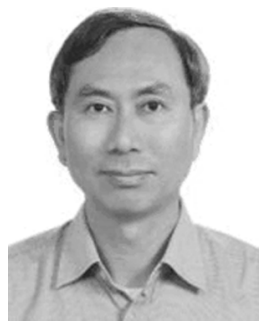

Shyh-Kang Jeng (M'86-SM'98) received the B.S.E.E. and Ph.D. degrees from National Taiwan University, Taipei, Taiwan, R.O.C., in 1979 and 1983, respectively.

In 1981, he joined the faculty of the Department of Electrical Engineering, National Taiwan University, where he is now a Professor. Between 1985 and 1993, he made numerous visits to the University of Illinois at Urbana-Champaign, as a Visiting Research Associate Professor and a Visiting Research Professor. In 1999, he spent six months at the Center for Computer Research in Music and Acoustics, Stanford University. His research interest includes numerical electromagnetics, ultrawideband wireless system, music signal processing, music information retrieval, intelligent agent applications, and electromagnetic scattering analysis. 